\title{
Qué proyectos STEM diseña y qué dificultades expresa el profesorado de secundaria sobre Aprendizaje Basado en Proyectos
}

\author{
Jordi Domènech-Casal \\ Servei d'Innovació i Formació, Departament d'Educació, Generalitat de Catalunya. Departament de \\ Didàctica de les Matemàtiques i les Ciències Experimentals, Universitat Autònoma de Barcelona. Barcelona. \\ España.jdomen44@xtec.cat \\ ORCID: https:// orcid.org/0000-0002-7324-0000

\begin{abstract}
Sílvia Lope
Centre de Recursos Pedagogics Especifics de Suport a la Innovació i la Recerca Educativa (CESIRE), Departament d'Educacio, Generalitat de Catalunya. Barcelona. España. Grup de Recerca Educativa en Ciències de la Salut (GRECS), Universitat Pompeu Fabra.Barcelona. España.slope@xtec.cat ORCID: https:// orcid.org/0000-0003-3192-1059
\end{abstract}

\section{Lluís Mora}

Centre de Recursos Pedagògics Especifics de Suport a la Innovació i la Recerca Educativa (CESIRE), Departament d'Educació, Generalitat de Catalunya.Barcelona.España.lmora1@xtec.cat ORCID: https:/ / orcid.org/0000-0002-5420-301X

[Recibido: 16 Noviembre 2018. Revisado: 28 Enero 2019. Aceptado: 8 Febrero 2019]

Resumen: El movimiento educativo STEM persigue incrementar las vocaciones científico-tecnológicas y conecta distintas herramientas, perspectivas y metodologías didácticas. El aprendizaje basado en proyectos (ABP) es una metodología privilegiada para la concreción de los objetivos STEM. Se ha llevado a cabo una formación a 82 docentes de secundaria (especialidades de Ciencias, Matemáticas, Tecnología) sobre ABP STEM, estructurada en dos módulos: presentación de buenas prácticas y diseño de propuestas con la ayuda de mentores. Se han analizado 87 proyectos diseñados por los participantes en relación a distintas componentes didácticas (Contexto, Conflicto, Discurso, Contenidos, Apertura, Interdisciplinariedad) y se han identificado 4 tipos principales de proyectos. Se analizan y discuten las percepciones del profesorado sobre las estrategias formativas y sus percepciones sobre dificultades y potencialidades de la metodología ABP en el despliegue STEM.

Palabras clave: Aprendizaje Basado en Proyectos; STEM; Educación Secundaria; Formación de profesorado

Which projects design and which difficulties express on Project-Based Learning Secondary Education teachers. Analysis of 87 project proposals

Abstract: The STEM proposal aims to promote Science and Technology careers and connects with several pedagogic tools, perspectives and methodologies. Project-Based Learning (PBL) is a candidate methodology for STEM development. We have set a training activity on PBL applied to STEM for 82 in-service teachers (Science, Technology and Mathematics teachers). This program has been structured in two modules: presentation of good practices and design of new projects with several scaffolds (canvas, mentoring). We have analysed 87 PBL proposals designed by the participants according several didactic components (Context, Conflict, Discourse, Contents, Openness, Interdisciplinary) and identified 4 main kinds of projects. We analyse and discuss perceptions of teachers on PBL applied to STEM and teacher-training strategies.

Keywords: Project-Based Learning; STEM; Secondary Education; Teacher-training

Para citar este artículo: Domènech-Casal J., Lope S., Mora L. (2019) Qué proyectos STEM diseña y qué dificultades expresa el profesorado de secundaria sobre Aprendizaje Basado en Proyectos. Revista Eureka sobre Enseñanza y Divulgación de las Ciencias 16(2), 2203. doi: 10.25267/Rev_Eureka_ensen_divulg_cienc.2019.v16.i2.2203 


\section{Introducción}

Informes de la Comunidad Europea han alertado de la disminución del interés del alumnado por las materias y carreras científico-tecnológicas en alumnado de primaria y secundaria en Europa (Rocard et al 2007), una dinámica que reproduce otras similares en países industrializados fuera de Europa, como EEUU. Las causas de esta falta de vocaciones son variadas, pero en el informe Rocard se identifican al menos dos. La primera causa está relacionada a la equidad y los roles de género: el alumnado femenino o de orígenes socioeconómicos humildes opta en muy bajas proporciones por profesiones científico-tecnológicas. La segunda es la enseñanza descontextualizada de la ciencia y la tecnología, que genera desinterés en los alumnos. Esto se ha conectado desde instituciones políticas a implicaciones industriales y económicas: la falta de vocaciones científico-tecnológicas puede tener a medio plazo consecuencias importantes en el tejido de innovación y desarrollo tecnológico. Además, este fenómeno conecta con perspectivas didácticas y políticas de la enseñanza de las ciencias.

Por un lado, las didácticas específicas reclaman una enseñanza de las ciencias más contextualizada y competencial (Sanmartí, Burgoa y Nuño 2011), en la que la participación del alumnado promueva su capacidad para aplicar, transferir y crear conocimiento científico. En esta línea se ubican las propuestas académicas e institucionales de la enseñanza de las ciencias mediante indagación y modelización (Caamaño 2011, Couso 2014, Windschitl, Thompson y Braaten 2008), el trabajo en Contexto (Blanco, España y Rodríguez 2012, Duranti y Goodwin 1992, Gilbert 2006) y el desarrollo de la competencia científica (OCDE 2013).

Por otro lado, se ha reclamado el papel de la enseñanza de las ciencias en formar ciudadanos competentes (Domènech-Casal 2018a). En este aspecto, son referentes las iniciativas alrededor de la alfabetización científica (Acevedo-Díaz 2004, Bybee 1997), la Educación Ambiental (Breiting 1999, Vilches Gil y Cañal 2010), la Ciencia, Tecnología y Sociedad -o CTS(Acevedo-Díaz, Vázquez y Manassero 2003, Aikenhead 2006, Hurd 1991, Yager 1996), las Controversias Socio- Científicas (Díaz y Jiménez-Liso 2012, Kolsto 2001, Sadler 2009), y la Investigación e Innovación Responsable (RRI, en sus siglas en inglés) (Domènech-Casal y Lope 2015).

\section{E1 "paraguas" STEM}

En conexión con este marco económico, didáctico y político, en los últimos años ha emergido en el espacio de la innovación educativa el término STEM, acrónimo de los términos en inglés Science, Technology, Engineering, Mathematics. El término STEM genera desconcierto entre profesionales y es usado con distintos significados dentro del mundo educativo (Akerson et al 2018, Bybee 2010, Brown et al 2011, Couso 2017, National Research Council 2014). Esto se debe a que, en realidad, el término no hace referencia directa a ninguna metodología, sino que se refiere al marco expuesto (en particular al económico) y cobija a todos los elementos educativos (precedentes o de nueva creación) que puedan ser de utilidad para su consecución. Por ello, podemos considerar que STEM no es en sí una metodología, sino un panel (variante y creciente) de herramientas tecnológicas, perspectivas pedagógicas y enfoques metodológicos (Couso 2017) que se ha considerado que pueden ser de utilidad para los objetivos STEM.

En la dimensión de herramientas tecnológicas, además de la programación/robótica, impresión 3D y el trabajo con sensores, suelen encontrarse como ítems STEM el trabajo con objetos tecnológicos accesibles como Apps y teléfonos móviles y aplicaciones de trabajo "Cloud" y Datos remotos (geolocalización, laboratorios virtuales, etc.) (Domènech-Casal 2014a y 2014b, López et al 2017). 
En la dimensión de perspectivas pedagógicas, el ámbito STEM recluta tres ejes, Inclusión, Creatividad y Ciudadanía. En el eje de Inclusión, STEM implica promover la participación y empoderamiento de las estudiantes en los ámbitos STEM, y la incorporación de estudiantes de familias de perfil socio-económico bajo. En el eje de la Creatividad, los espacios de creación prospectiva, como el Tinkering (Simarro y Couso 2016), o la integración de las Artes (en un sentido amplio) en la enseñanza de la Ciencia, Tecnología y Matemáticas, lo que ha venido a llamarse STEAM (Henriksen 2014). En el eje de la Ciudadanía, podríamos incluir el trabajo con Controversias Socio-Científicas, (Sadler 2009), la Ciencia Ciudadana (Bonney et al 2009), la Investigación e Innovación Responsable (RRI) (Domènech-Casal 2018a) y la Educación Ambiental o Educación para el Desarrollo y la Paz (Mesa 2000, Gil y Vilches 2001).

En la dimensión de enfoques metodológicos la llegada de STEM a la educación ha coincidido con una revitalización de metodologías activas específicas y generales. Lo importante ya no es sólo saber ciencias, matemáticas y tecnología, sino saber resolver problemas en contextos reales "pensando como" matemáticas/os, científicas/os e ingenieras/os. Por ello, algunos referentes metodológicos propios de las áreas STEM y sus discursos epistémicos se cobijan también dentro del gran paraguas STEM. En matemáticas, la resolución de problemas, entendida en sentido epistémico, no algorítmico, es decir abordar problemas complejos para definir una estrategia matemática de resolución (Pólya 1962, Schoenfeld 1992, Simarro 2016). En ciencias, la indagación y la modelización (Bybee 1997, Caamaño 2011), o propuestas como la IBSE (Llewellyn 2005), que proponen la enseñanza de las ciencias emulando el proceso científico de creación del conocimiento (observar evidencias, diseñar experimentos, conectar con -y elaborar- modelos...). En tecnología, el conocido como Proceso Tecnológico, que en contacto con el mundo de la innovación industrial y el Diseño ha dado lugar a distintas aproximaciones, como el Design Thinking, el fenómeno "Maker" o el "Learning by Design" (Albalat 2017, Bordignon, Iglesias y Hahn 2016). La orientación a contextos implica también la necesidad de perspectivas interdisciplinarias, en la línia de lo propuesto como educación CTS (Ciencia, Tecnología y Sociedad) (Aikenhead y Ryan 1992). Por otro lado, este cambio metodológico también ha reivindicado objetivos didácticos como las "soft skills", habilidades o capacidades necesarias, como el trabajo cooperativo, o el Pensamiento Computacional, que tienen aplicación en las distintas áreas STEM (Brennan y Resnick 2012, Simarro et al 2016 Wing 2006).

\section{El ABP, una metodología privilegiada}

El Aprendizaje Basado en Proyectos (ABP) es una metodología didáctica de una cierta antigüedad, que ha cobrado un especial interés en los últimos años (Domènech-Casal 2016, Sanmartí 2016), en la que el contexto juega un papel importante y que incluye una amplia diversidad de enfoques (Grau 2009). Propuesto por Kilpatrick en su opúsculo "The Project Method" (1918), el ABP se basa en la necesidad de un propósito para que se produzca un aprendizaje relevante. Dentro del ABP, Kilpatrick incluye 4 categorías de proyectos, según el propósito que los anima, y que podríamos resumir en: Elaborar un producto, Resolver un problema, Disfrutar de una experiencia estética y Obtener un conocimiento. Estas 4 categorías pueden agruparse en dos grupos (Domènech-Casal 2018b, 2019). Por un lado, la categoría orientada a Obtener un conocimiento -lo que podríamos llamar Proyectos de Aprendizaje- en la que no existe un propósito externo a obtener conocimiento. En la Enseñanza de las Ciencias, corresponderían a esta orientación las aproximaciones de Indagación y Modelización (Windschitl, Thompson y Braaten 2008), explícitamente orientadas al propósito de conocer el modelo científico que rige un sistema. El otro grupo lo conforman las otras tres categorías, que tienen en común que el eje de la actividad del alumnado es un "propósito" u objetivo externo al aprendizaje. Este grupo, y en particular, las categorías de Elaborar un Producto o Resolver un Problema, es el que inspira las formulaciones actuales del ABP (Larmer, 
Mergendoller y Boss 2015). Basa su fundamento pedagógico en la idea constructivista según la cual los aprendizajes son más profundos y transferibles cuando se realizan de manera instrumental en la resolución de un conflicto y la necesidad de que los aprendices tengan un papel activo que ejercen en comunidad. Esta idea se concreta en varios tipos de ABP, que incluyen por ejemplo los Estudios de Caso Dirigido (Domènech-Casal 2017a), el Design Thinking (Albalat 2017) o las Controversias Socio-Científicas (Sadler 2009). Esta orientación a un propósito conjuga bien con las necesidades STEM y también conecta el ABP con las propuestas de algunos autores sobre la necesidad de ubicar el aprendizaje de las ciencias en contextos relevantes (Gilbert 2006, Sanmartí y Márquez 2017).

Aun así, varios autores advierten de la necesidad de tener en cuenta particularidades metodológicas del ABP en el diseño de actividades (Domènech-Casal 2019, Grau 2009, Sanmartí y Márquez 2017): 1) La relación Conflicto-Contexto-Contenido: La actuación/resolución de un conflicto en un contexto suele "reclutar" más de un contenido (lo que acostumbra a vincular el ABP a la interdisciplinariedad) y lo habitual es que la resolución del conflicto no requiera el dominio completo del contenido, sino sólo un dominio parcial. 2) El discurso propio de las áreas y la interdisciplinariedad: como hemos dicho, cada área tiene su propio discurso, y su participación puede ser mayor o menor, lo que implica distintos niveles de desarrollo epistémico. La conjugación de los discursos de área con la interdisciplinariedad es compleja. 3) La Apertura: la capacidad que tiene el alumnado de tomar decisiones en el proyecto, que puede ir desde decidir el propósito (muy abierto), tomar decisiones estratégicas en un marco global propuesto por el docente (estructurado) o tomar pequeñas decisiones en una secuencia de tareas pre-establecida (cerrado).

Si bien hay informes que indican que en la enseñanza de áreas STEM la colaboración entre disciplinas y docentes y las metodologías activas correlacionan con el éxito educativo (Clavel, Méndez y Crespo 2016), son necesarios marcos que permitan distinguir los distintos tipos de ABP y sus características didácticas para poder evaluar el impacto de cada tipo de ABP en el aprendizaje de las Matemáticas, las Ciencias y la Tecnología.

\section{La formación del profesorado en ABP}

Las propuestas ABP generan desconcierto por falta de definición y especificación de las características de cada tipo de ABP (Santmartí 2016), también en la formación del profesorado. La transición hacia metodologías ABP requiere un acompañamiento y andamiaje que permita identificar los elementos didácticos que deben articularse en las propuestas didácticas (Domènech-Casal 2016, Ertmer y Simons 2005). Los programas de despliegue y formación de profesorado STEM adquieren formas muy diversas, incluyendo talleres, cursos y mentores, y difieren mucho en la duración, intensidad y materiales y apoyo proporcionado (Smith y Ingersoll 2004). Existen algunas investigaciones incipientes sobre el efecto de estas modalidades en los cambios en percepciones y prácticas de los docentes. Estas investigaciones indican que el profesorado percibe como cruciales aspectos como ubicar el aprendizaje en un problema o analizar qué contenidos se trabajan realmente en una actividad STEM (Wang, Moore, Roehrig y Park 2011) o el dominio del contenido propio de las áreas implicadas (Blomeke, Suhl, y Kaiser 2011). También se ha descrito como factor positivo en las propuestas formativas de profesorado STEM la presencia de un proyecto marco (un portfolio o artículo personal de investigación), proveer experiencias prácticas (Wilson 2009), la coherencia de la propuesta y la orientación al desarrollo de habilidades (Garet, Porter, Desimone, Birman y Yoon 2001). De todos modos, algunos metaanálisis indican que es difícil identificar qué elementos de la formación de profesorado (formato, duración, apoyos...) tiene más impacto en el ulterior aprovechamiento profesional (Desimone, Porter, Garet, Yoon y Birman 2002, Luft, Roehrig y Patterson 2003). 
El impulso de innovación del ámbito STEM es una oportunidad para la enseñanza de las ciencias. No obstante, se requieren herramientas formativas que permitan acompañar el diseño y analizar la arquitectura didáctica y aportación real de las actividades ABP STEM.

\section{Objetivos}

Los objetivos de este artículo son:

1) Desarrollar elementos de apoyo para la formación de profesorado sobre ABP STEM.

2) Analizar los proyectos ABP STEM que diseña el profesorado de secundaria.

3) Analizar percepciones y necesidades formativas del profesorado en relación al diseño, aplicación y eficacia de secuencias ABP STEM.

\section{Metodología}

Se organizó una formación de profesorado en activo sobre STEM y ABP. En la formación participaron un total de 82 docentes de secundaria (33 de ciencias, 22 de tecnología y 27 de matemáticas). La formación se estructuró del modo siguiente: se realizaron 4 sesiones, cada sesión con dos módulos. En el primer módulo distintos ponentes expertos en la metodología presentaban 4 experiencias ABP STEM aplicadas en el aula y en el segundo los participantes -en grupos de 5-6 participantes- ayudados por mentores expertos y el soporte de una plantilla (disponible para su descarga en el blog de la formación ${ }^{1}$ ) colaboraron diseñando propuestas didácticas de proyectos. La plantilla consta de distintos apartados en los que el profesorado explicita el Contexto, los Contenidos, la secuencia y apoyos y narra el desarrollo de la propuesta en distintos aspectos (evaluación, organización social, apertura...). Las dos primeras sesiones se orientaron a proyectos de área (Matemáticas, Ciencias o Tecnología), la tercera a proyectos interdisciplinarios y la última a la defensa y discusión de propuestas generadas por los participantes. El contenido de las ponencias fue sintetizado y está disponible en distintos artículos publicados por los ponentes de proyectos de Ciencias (Marchán-Carvajal et al 2017, Touron et al 2017) Matemáticas (Margelí et al 2017, Planella et al 2017), Tecnología (Josa et al 2017, Villar et al 2017) e Interdisciplinares (Redondo et al 2017).

\section{Análisis de propuestas de proyectos elaboradas por los participantes}

El profesorado participante entregó como actividades evaluables varias propuestas de proyectos elaboradas en las distintas sesiones a lo largo de la formación según la plantilla propuesta, sumando un total de 87 proyectos disponibles en el blog de la formación.

Para la evaluación de los propuestas de proyectos se utilizó la rúbrica ABPMap (descargable en: https://bit.ly/2BcXRLd). Esta rúbrica, descrita en anteriores trabajos (Domènech-Casal 2018b) incluye el análisis de 6 componentes didácticas (Contexto, Conflicto, Discurso, Contenidos, Apertura, Interdisciplinariedad) relacionadas con lo expuesto en la introducción y distintos niveles para cada una de las componentes. Se seleccionó esta rúbrica para este estudio por haber sido testada en la evaluación de proyectos aplicados en el aula y referir de modo concreto a las complejidades didácticas descritas en la introducción para el ABP.

Los proyectos fueron evaluados con la rúbrica estableciendo una puntuación (1-4) para cada componente (como se describe en la rúbrica), elaborando el perfil de componentes de cada proyecto (la combinación de puntuaciones de cada proyecto). Para la representación de los datos, se elaboró el gráfico ABPMap: se ubicaron las componentes en filas y se representó el número de proyectos que se situaban en cada puntuación $(1 \rightarrow 4)$. Se identificaron con códigos

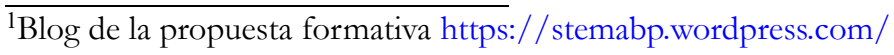


de colores las puntuaciones con más proyectos, de forma similar a un mapa de curvas de nivel (Fig. 1).

Por otro lado, los proyectos fueron sometidos a un análisis de clúster jerárquico (Johnson 1967, Jardine y Sibson 1971) destinado a determinar si ciertos perfiles de componentes eran frecuentes y permitían identificar tipologías de proyectos. Para ello, se siguieron los pasos siguientes:

1) Se eligieron 15 proyectos al azar y se calculó para cada uno de ellos cuántos proyectos del total (87 proyectos) tenían un perfil de componentes similar que se diferenciase como máximo acumulado en un total de 3 puntos.

2) Se seleccionó el proyecto que tenía más proyectos similares y se definió el grupo como Tipo A.

3) Se eliminaron del listado los proyectos de tipo A.

El proceso se repitió varias veces hasta generar los tipos B, C, y D. Los proyectos restantes no pudieron ser agrupados, pues la diversidad de perfiles no permitió formar clústers más allá de 4 proyectos, que se consideraron no representativos. Para descartar el efecto del azar en la elección inicial de los 15 proyectos, se repitió dos veces el proceso desde el principio, confirmando los mismos resultados. Se representaron sobre el ABPMap los tipos de proyectos identificados (Fig. 2).

\section{Análisis de percepciones y valoraciones de los participantes}

Los participantes completaron como cierre de cada sesión un portfolio individual, que incluyó los dos apartados siguientes: "Me anoto, me interesa", "No entiendo, no veo, me falta", en los que anotaron en breves frases sus valoraciones. La interpretación del contenido se ha realizado a través del proceso de clasificación sistemática de codificación e identificación de temas o patrones (Hsieh y Shannon 2005). En primer lugar se procedió a un análisis descriptivoinductivo de las respuestas, se organizaron en categorías y se contabilizó el número total de aportaciones en cada categoría (ejemplos de respuestas están disponibles en: https://bit.ly/2PZkLho).

Los participantes valoraron también en una encuesta -mediante una escala Likert $(1 \rightarrow 6)$ - su grado de apoyo a la importancia de distintos elementos didácticos (evaluación, recursos...) en relación a fortalezas y debilidades del ABP. Se calcularon las puntuaciones medias y la desviación típica para cada uno de los elementos, que se enuncian en los resultados.

Por distintas razones de orden práctico pudieron recogerse un total de 55 portfolios (21 de profesores de ciencias, 13 de profesores de tecnología y 21 de matemáticas) y 56 encuestas. La edad de los participantes se situaba en las franjas de 30-40 y 40-50 (36 y 39\%, respectivamente), y entre los $20-30$ y $50-60$ que representaban porcentajes menores $(5 \%$ y $20 \%$ ). En lo relativo a la experiencia docente, un $27 \%$ tenía menos de 5 años de experiencia. Un $21 \%$ tenía entre 5 y 10 años, un $27 \%$ entre 10 y 15 años y el resto tenía más de 15 años de experiencia. De todos ellos, un 52\% se declaraba inexperto en el ABP, un $7 \%$ se declaraba experto y el resto declaraba haberse iniciado en la práctica del ABP. Por materias, los participantes en la encuesta eran de Matemáticas (37\%), Ciencias Experimentales (45\%), Tecnología $(16 \%)$ y el resto de otras especialidades. 


\section{Resultados}

\section{Análisis de los proyectos elaborados por los participantes}

Se colectaron 87 diseños de proyectos durante las distintas sesiones. Los distintos proyectos presentaron una distribución desigual en las distintas componentes (Fig. 1). Sólo 9 de los 87 proyectos incorporaron 3 o más materias, y 58 (un 75\%) incorporaron sólo una materia (algo esperable, pues dos de las sesiones se dedicaron al desarrollo de proyectos de área). Los proyectos se concentran en una zona con un despliegue moderado (nivel 2) del conflicto, los contenidos y el grado de apertura. Principalmente se crearon proyectos en los que los contenidos están en la periferia del conflicto, se desarrollan de manera parcial e informal y los alumnos participan en la evaluación, pero no en decisiones sobre los productos.

Al realizar el análisis de clusters, se identificaron en el grupo de 87 proyectos 4 clusters correspondientes a distintos perfiles de niveles de desarrollo de las componentes ABP STEM. Los perfiles de componentes de cada cluster se describen a continuación y se representan en la Fig. 2.

Proyectos de tipo A: Proyectos de una sola área, que emergen del mundo real, del que incorporan agentes. Los contenidos están en el núcleo del conflicto y son esenciales (pero no suficientes) para resolverlo. Se aplican procesos y formatos propios de creación de conocimiento de las áreas STEM. Los contenidos son desarrollados de forma parcial y formalizados activamente. Los alumnos no participan en la planificación, pero sí se auto-evalúan.

Un ejemplo de este tipo de proyectos es el proyecto P11, titulado "Contaminación en el Iseo", un Estudio de Caso Dirigido para $3^{\circ}$ de ESO en el que el alumnado toma el rol verosímil de integrantes de una ONG y deben, a partir de un escenario concreto, determinar qué puntos del recorrido de un río deben analizar en el laboratorio, realizar los análisis y establecer la causa de la contaminación en un informe final en formato científico.

Proyectos de tipo B: Incorporan materiales del mundo real, pero el contexto y rol del alumnado no es verosímil. Los contenidos -de una sola área- no juegan un papel significativo en la resolución del conflicto. Se aplican de forma pautada procesos de obtención de datos propios de las áreas STEM. Los contenidos se aplican y desarrollan de manera parcial y no formalizados. Consisten en una sucesión de tareas cerradas. Un ejemplo de este tipo de proyectos es el P10, "Construcción de un árbol de navidad geométrico" en el que los alumnos deben usar figuras geométricas con materiales pautados (cartulina y cañas de refresco) para construir árboles de navidad para la decoración del instituto. 
Proyectos de tipo C: Proyectos Interdisciplinarios que incorporan 3 áreas STEM. El proyecto tiene sentido sólo dentro del aula y no incorpora formatos del mundo real. El conflicto puede resolverse sin los contenidos, que son reproducidos sin usar ninguna aproximación específica de áreas STEM. Los alumnos no participan en la planificación, pero sí se auto-evalúan. Un ejemplo es el proyecto 48, "Escape Room" en el que los alumnos deben formar equipos y crear un conjunto de pruebas de preguntarespuesta o resolución de

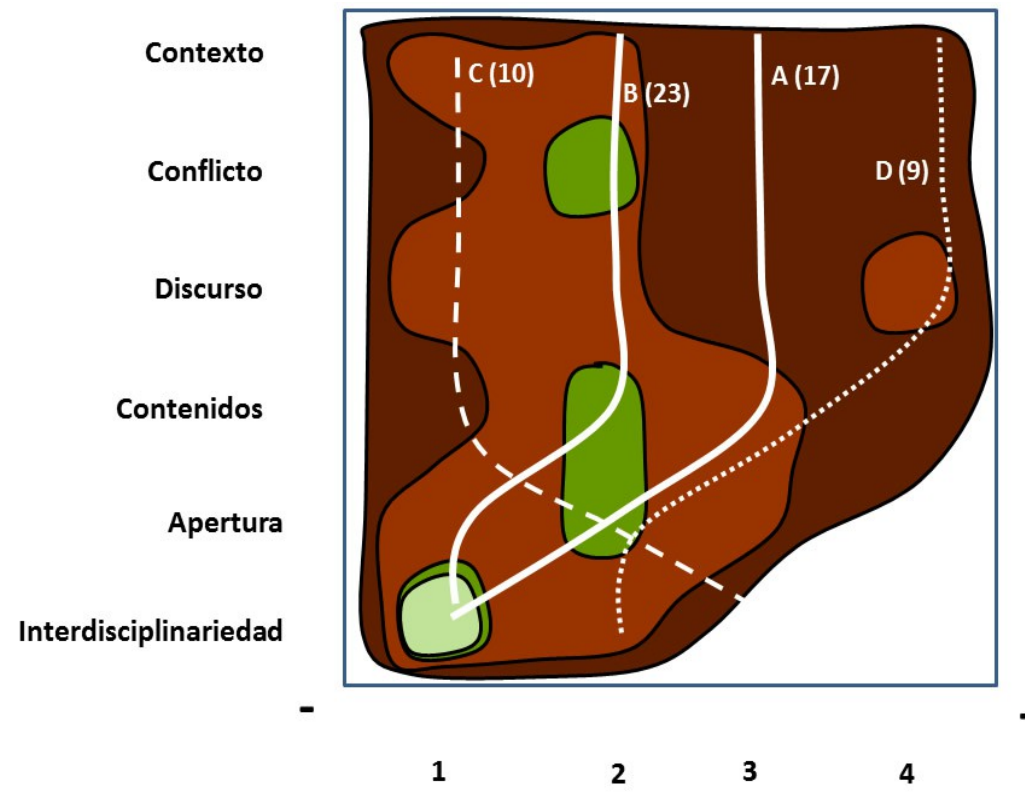

Figura 2. Representación bidimensional de los 4 Clusters (A, B, C y D) de proyectos identificados sobre el Mapa ABP. Entre paréntesis la cantidad de proyectos de cada tipo.

\section{algoritmos que abarquen distintas áreas STEM para que sea resuelto por otro equipo.}

Proyectos de tipo D: Proyectos en los que participan dos disciplinas y se incorporan elementos del mundo real y los contextos y roles son reales. Los contenidos se identifican con el núcleo del conflicto y son esenciales para su resolución. Se desarrollan dinámicas epistémicas propias de las STEM para la creación y evaluación de conocimiento. Los contenidos son construidos de forma parcial y formalizados. Los alumnos no participan en la planificación, pero sí se auto-evalúan.

Un ejemplo de este tipo de proyectos es el proyecto P73 dirigido a $3^{\circ}$ de ESO, "Diseño de un cosmético", en el que cada equipo es una empresa de cosméticos que debe usar conocimientos de ciencias y tecnología para elaborar su producto, que presentan a representantes de empresas de farmacia y cosmética.

28 de los 87 proyectos no pudieron agruparse.

\section{Resultados de portfolios y encuesta al profesorado}
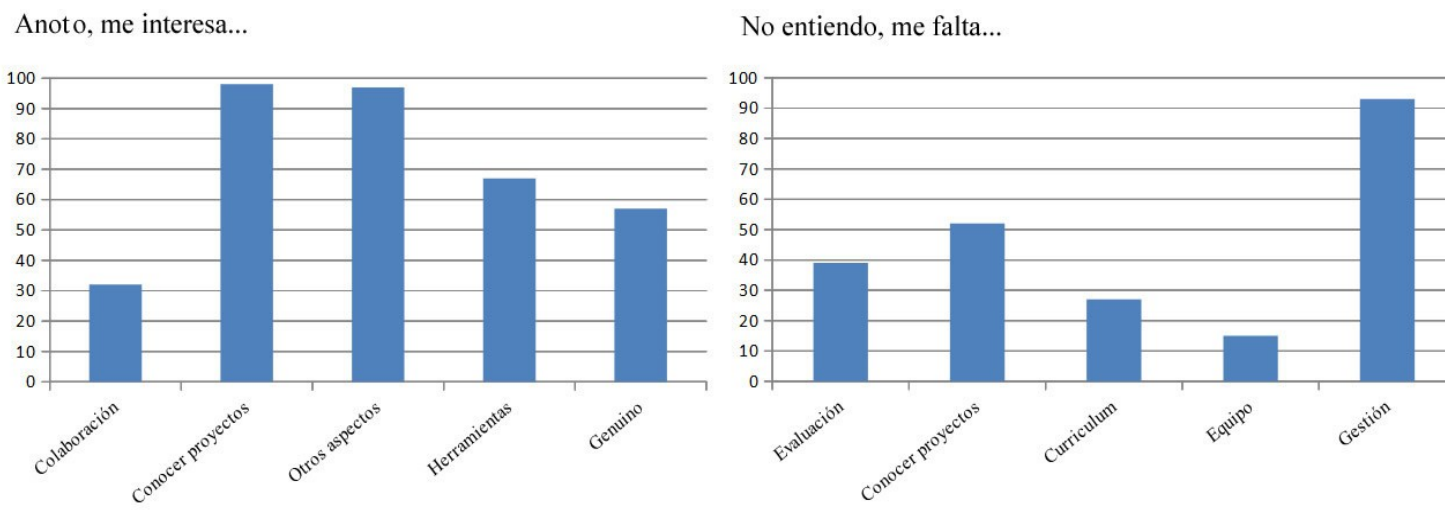

Figura 3. Número total de aportaciones en cada categoría para los apartados del portfolio "Me anoto, me interesa" y "No entiendo, no veo, me falta". 
El análisis de los portfolios realizados por los participantes a lo largo de las jornadas ha permitido obtener una imagen de su percepción sobre distintos aspectos del ABP, que se han cuantificado por categorías (Fig. 3), destacando el interés de conocer proyectos, herramientas, y la contextualización real del aprendizaje (Genuino) de los ABP y las dificultades en la Gestión.

En la encuesta, la evaluación, logística y la falta de espacios de coordinación fueron los más puntuados como posibles dificultades en la aplicación del ABP (Fig. 4a).

El desarrollo de las habilidades de trabajo en equipo es el aspecto que más destaca el profesorado como fortaleza del ABP (Fig. 4b). Observando las desviaciones tipo se detecta que el profesorado detecta de forma distinta las dificultades del ABP, pero es más homogéneo en la detección de sus fortalezas. El profesorado considera que son elementos de calidad de una propuesta $\mathrm{ABP}$ que se evalúen los aprendizajes, que tenga sentido en el mundo real y que se trabaje en equipo (Fig. 5a).
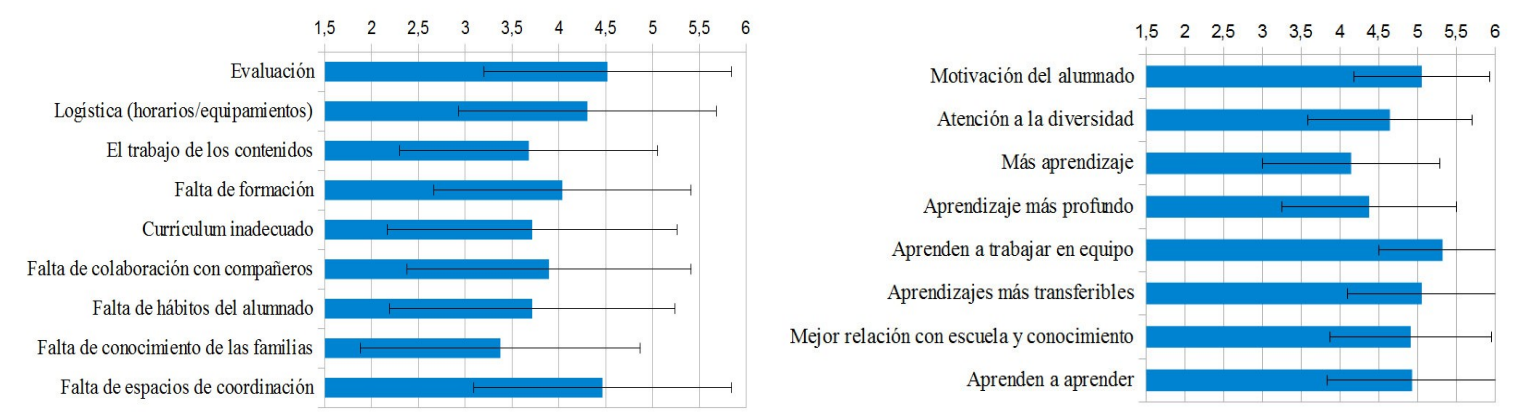

Figura 4. a) Media y desviación típica de hasta qué punto considera el profesorado que cada uno de los elementos es una dificultad para el ABP (6: muy de acuerdo; 1 muy en desacuerdo). b) Valores medios y desviación tipo de la valoración que hace el profesorado de distintas proposiciones como fortalezas de la metodología.

Al preguntar al profesorado sobre los enfoques para la formación de profesorado en ABP de forma genérica -no sólo en lo referido a la formación en la que participa- el profesorado propone distintos enfoques o estrategias (Fig. 5b). La desviación tipo se mantiene baja en los distintos ítems (alrededor de 1), lo que sugiere que, a pesar de identificar distintas dificultades, el profesorado tiene visiones similares sobre qué tipo de enfoques formativos del profesorado pueden ayudar a resolverlas.

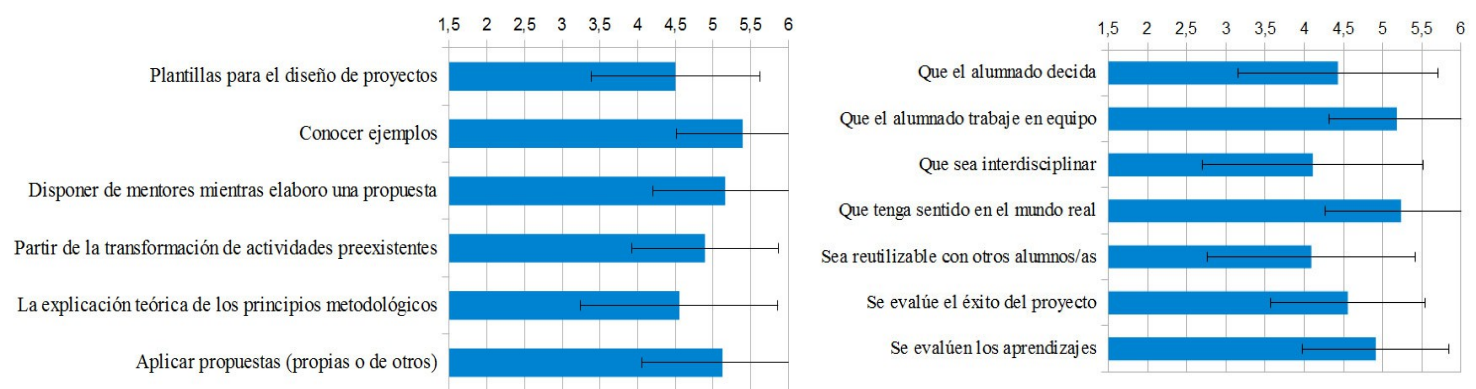

Figura 5. a) Valores medios y desviación tipo de la valoración que hace el profesorado de elementos de calidad de una propuesta ABP. b) Valores medios y desviación tipo de la valoración que hace el profesorado de la utilidad de apoyos/experiencias formativas para el desarrollo de prácticas ABP. 


\section{Discusión}

El diseño de la formación promovió el diseño de distintos materiales de apoyo para la formación ABP en STEM (la plantilla de diseño de proyectos, el portfolio, los artículos con la síntesis de las ponencias) y su análisis didáctico (la rúbrica ABP Map). Todos ellos están disponibles para su descarga y más de un año después de la formación, los datos de cibermetría de los servidores nos indican que continúan siendo descargados y tenemos constancia de que han sido adoptados como herramientas en otras formaciones independientes.

\section{Componentes didácticas en el diseño de proyectos STEM}

La mayoría de los proyectos analizados sitúan a los contenidos en la periferia del conflicto (no juegan un papel fundamental en su resolución) y el alumnado tiene poca participación en su planificación. Esto se contradice con dos de las motivaciones que suelen esgrimirse en la adopción del ABP como metodología, esto es, el aprendizaje instrumental y la creatividad y autonomía del alumnado (Sanmartí y Márquez 2017). Una gran parte de los proyectos propuestos por el profesorado puede explicarse en base a 4 perfiles que hemos identificado. No hay una razón aparente de por qué estos perfiles son tan abundantes en la muestra, pero parecen indicar visiones del profesorado sobre qué es un proyecto o "zonas de equilibrio", espacios didácticamente posibles o adaptables a la logística (espacios, currículum) o liturgias (evaluación, roles de profesorado y alumnado) de las aulas. En los 4 perfiles, hay una asociación en el despliegue de las componentes Contexto, Conflicto y Discurso. Esto parece indicar que el nivel de despliegue de una de estas componentes puede favorecer el despliegue de las otras. Inesperadamente, en estos 4 perfiles el despliegue de la interdisciplinariedad es opuesto al contexto, contradiciendo la visión intuitiva de que en el diseño de proyectos la interdisciplinariedad es una consecuencia de la ubicación del proyecto en contextos más reales (Grau 2009). Esta tendencia sólo se rompe con los proyectos de tipo D, que son los que desarrollan más las componentes de Contexto, Conflicto, Discurso y Contenidos.

Es importante hacer constar que este análisis se ha realizado con proyectos diseñados (no aplicados), y que es común que los proyectos experimenten modificaciones en su aplicación, por lo que nuestras interpretaciones se limitan al diseño de los proyectos.

\section{Percepciones del profesorado en relación al diseño de secuencias ABP STEM y necesidades formativas}

El profesorado participante destaca, en los portfolios y encuestas, como valores positivos de la metodología ABP que mejora la motivación y el compromiso personal, y la autonomía (aun así, sólo en 26 de los proyectos presentados el alumnado participa de manera significativa en la planificación, y ninguno de los 4 perfiles identificados lo hace). Los resultados ponen de manifiesto una importante preocupación en relación a cómo evaluar a los alumnos. Existe un conflicto entre la evaluación del grupo y la individual, ya que efectivamente es complicado tener una visión individualizada del aprendizaje a partir de productos colectivos, y también entre la evaluación del proceso o del producto final. A algunos profesores también les preocupa organizar el centro o poder cumplir el currículum trabajando siempre por proyectos, esto se detecta tanto en los portfolios como en la encuesta. Sin embargo, algunas experiencias muestran que sí es posible trabajar mediante proyectos la totalidad del currículo y que las objeciones a estas metodologías por razones de tiempo no tienen fundamento (DomènechCasal 2017c, Trujillo 2016).

Al ser preguntado sobre los enfoques en formación de profesorado, las estrategias de formación/apoyo más valoradas por el profesorado han sido aquéllas más vinculadas a la 
práctica (conocer ejemplos, aplicar propuestas y disponer de mentores en la práctica), algo ya descrito por otros autores (Ertmer y Simons 2005). Los participantes han destacado además la importancia de conocer proyectos reales que propongan situaciones de aprendizaje auténticas (vinculadas a contextos reales), y los aspectos técnicos relativos a la evaluación y la autonomía del alumnado. En general, el profesorado presenta mayor consenso en relación a las fortalezas pedagógicas y necesidades formativas del ABP que en relación a las dificultades identificadas para su aplicación.

\section{Conclusiones}

Del análisis de nuestros resultados, y con la reserva de las limitaciones que entrañan (muestra reducida, proyectos no aplicados) extraemos las siguientes conclusiones y orientaciones:

1) Existe una discrepancia entre lo que el profesorado afirma perseguir al adoptar el ABP (aprendizaje instrumental y profundo, autonomía del alumnado...) y las características de los proyectos que acaba diseñando, la mayoría de los cuales desarrollan poco aspectos como el Conflicto, el Discurso, los Contenidos o la Apertura. Esto hace necesario que antes de aplicar un ABP en el aula se analice lo que promueve o no con herramientas evaluadoras similares o equivalentes al ABPMap.

2) Se han podido identificar 4 perfiles distintos de proyectos ABP STEM que pueden corresponderse a fórmulas didácticas paradigmáticas. Consideramos que puede ser de gran interés confirmar esos perfiles en muestras más grandes de proyectos aplicados en el aula y relacionarlas con su eficacia en el desarrollo de la Competencia científica: ¿de confirmarse, son los proyectos de tipo D una fórmula privilegiada? En este sentido, nos proponemos analizar una nueva muestra de otros 120 proyectos ABP STEM diseñados por profesorado en activo y en formación inicial e invitamos a otros investigadores al uso de ABPMap.

3) Contrariamente a lo esperado, la Interdisciplinariedad no va necesariamente asociada a un mejor desarrollo del Contexto. En los perfiles identificados, el perfil con mayor interdisciplinariedad es el que menores valores aporta de desarrollo de Discurso y Conflicto, desde nuestro punto de vista fundamentales para la Competencia científica. De ello extraemos que es necesario ser cautelosos con la asunción generalizada de que la interdisciplinariedad redunda por sí misma en un mayor valor competencial de las actividades de enseñanza y, en particular, del ABP.

4) El profesorado da más valor a las acciones formativas más vinculadas a la práctica real (como el papel de mentores, aplicar proyectos preexistentes y conocer ejemplos reales proyectos aplicados) que a otros recursos (plantillas, marcos teóricos). Aunque cumplir esta preferencia no implica necesariamente que ello redunde en mejores propuestas formativas, sí recomienda que las acciones formativas ABP STEM se hagan en complicidad y conexión muy cercana con centros educativos o docentes expertos que estén desarrollando esas prácticas.

5) Además de su uso para el análisis extensivo de propuestas ABP STEM, la rúbrica ABPMap permite su uso para la evaluación y mejora de proyectos. Aún sin ser necesario desplegar todas las componentes en su valor máximo, puede ser de utilidad como indicadora de vías de mejora: ¿puede este proyecto beneficiarse de una mejor contextualización? ¿juegan realmente los contenidos un papel este proyecto? 


\section{Agradecimientos}

Los autores agradecen al profesorado participante y las siguientes instituciones colaboradoras en la organización de las Jornadas STEM-ABP su apoyo: CESIRE (Departament d'Ensenyament de la Generalitat de Catalunya) y Fundació Catalana per a la Recerca i la Innovació (FCRI). Reflexiones incluidas en este artículo se enmarcan en la reflexión metodológica del grupo de investigación consolidado LICEC (referencia 2014SGR1492) por AGAUR y financiado por el Ministerio de Economía y Competitividad (referencia EDU2015-66643-C2-1-P) y el despliegue del Plan STEAMCat del Departament d'Ensenyament de la Generalitat de Catalunya, con el apoyo de Digna Couso y Ana Albalat, a quienes agradecemos su orientación y reflexiones.

\section{Referencias}

Acevedo-Díaz J.A. (2004) Reflexiones sobre las finalidades de la enseñanza de las ciencias: educación científica para la ciudadanía. Revista Eureka sobre Enseñanza y Divulgación de las Ciencias, 1(1), 3-16.

Acevedo-Díaz J.A., Vázquez A. y Manassero, M.A. (2003) Papel de la educación CTS en una alfabetización científica y tecnológica para todas las personas. Revista Electrónica de Enseñanza de las Ciencias, 2(2), 80-111.

Akerson V.L., Burgess A., Gerber A., Guo M. (2018) Disentangling the meaning of STEM: Implications for Science Education and Science Teacher Education. Journal of Science Teacher Education, 29(1), 1-8.

Aikenhead G.S. y Ryan A.G. (1992) The development of a new instrument: 'Views on Science-Technology-Society' (VOSTS). Science Education, 5 (76), 477-491.

Aikenhead G.S. (2006) Science Education for Everyday Life. Evidence-Based Practice. New York: Teachers College Press.

Albalat A. (2017). Design thinking en STEAM. Revista Ciències, 34, 29-34.

Blanco A., España E. y Rodríguez F. (2012) Contexto y enseñanza de la competencia científica. Alambique, Didáctica de las Ciencias Experimentales, 70, 9-18.

Blömeke S., Suhl U. y Kaiser G. (2011) Teacher education effectiveness: Quality and equity of future primary teachers' mathematics and mathematics pedagogical content knowledge. Journal of Teacher Education, 62, 154-171.

Bonney R., Cooper C. B., Dickinson J., Kelling S., Phillips T., Rosenberg K.V. y Shirk J. (2009) Citizen Science: A Developing Tool for Expanding Science Knowledge and Scientific Literacy. BioScience, 59 (11), 977-984.

Bordignon F.R.A., Iglesias A.A. y Hahn A. (2016) Prácticas maker en la Escuela Secundaria. Comunicación y pedagogia, 291-292, 72-79

Breiting S. (1999) Hacia un nuevo concepto de educación ambiental. En: 30 Reflexiones sobre educatión ambiental. Madrid: Ministerio de Medio Ambiente.

Brennan K. y Resnick K. (2012) New frameworks for studying and assessing the development of computational thinking. Proceedings of the 2012 annual meeting of the American Educational Research Association (pp.1-25).Vancouver. [http://web.media.mit.edu/ kbrennan/files/Brennan_Resnick_AERA2012_CT.pdf]

Brown R., Brown J., Reardon K. y Merrill C. (2011) Understanding STEM: Current perceptions. Technology and Engineering Teacher, 70(6), 5-9.

Bybee R.W. (1997) Acbieving scientific literacy: From purposes to practices. Portsmouth: Heinemann. 
Bybee R.W. (2010a) Advacing STEM Education: A 2020 Vision. Technology and Engineering Teacher, 70(1), 30-35.

Bybee R.W. (2010b) What is STEM Education? Science, 329 (5995), 996-996.

Caamaño A. (2011) Contextualización, indagación y modelización. Tres enfoques para el aprendizaje de la competencia científica en las clases de química. Aula de Innovación Educativa, 207, 17-21.

Clavel J.G., Méndez I. y Crespo F.J.G. (2016) Are teacher characteristics and teaching practices associated with student performance? TIMMS Policy Brief. http://pub.iea.nl/fileadmin/user_upload/Policy_Briefs/IEA_Policy_Brief_Sep2016.p df

Couso D. (2014) De la moda de "aprender indagando" a la indagación para modelizar: una reflexión crítica. XXVI Encuentro de Didáctica de las Ciencias Experimentales. Huelva (Andalucía).

Couso D. (2017) Perquè estem a STEM? Definint l'alfabetització STEM per a tothom i amb valors. Revista Ciències, 34, 21-29.

Desimone L., Porter A.C., Garet M., Yoon K.S. y Birman B. (2002) Effects of professional development on teachers' instruction: Results from a three-year longitudinal study. Educational Evaluation and Policy Analysis, 24, 81-112.

Díaz N. y Jiménez-Liso M. R. (2012) Las controversias sociocientíficas: temáticas e importancia para la educación científica. Revista Eureka sobre Enseñanza y Divulgación de las Ciencias, 9(1), 54-70.

Domènech-Casal, J. (2014a). Indagación en el aula mediante actividades manipulativas y mediadas por ordenador. Alambique, Didáctica de las Ciencias Experimentales, 76, 17-27.

Domènech-Casal J. (2014b) Contextos de indagación y controversias socio-científicas para la enseñanza del Cambio Climático. Enseñanza de las Ciencias de la Tierra, 22(3), 267-276.

Domènech-Casal J. (2016) Apuntes topográficos para el viaje hacia el \#ABP. Cuadernos de Pedagogía, 742, 59-62.

Domènech-Casal J. (2017a) Aprendizaje Basado en Proyectos y competencia científica. Experiencias y propuestas para el método de estudios de caso. X Congreso Internacional sobre Investigación en Didáctica de las Ciencias, Sevilla 2017.

Domènech-Casal J. (2017b) Aprenentatge Basat en Projectes en àmbits STEM. Claus metodològiques i reptes. Revista Ciències, 33, 2-7.

Domènech-Casal J. (2017c) Proyectando BioGeo, un itinerario de trabajo por proyectos contextualizados basado en la indagación y la Naturaleza de la Ciencia. Alambique, Didáctica de las Ciencias Experimentales, 89, 54-61.

Domènech-Casal J. (2018a) Comprender, Decidir y Actuar: una propuesta-marco de Competencia Científica para la Ciudadanía. Revista Eureka sobre Enseñanza y Divulgación de las Ciencias, 15 (1), 1105.

Domènech-Casal J. (2018b) Aprendizaje Basado en Proyectos en el marco STEM. Componentes didácticas para la Competencia Científica. Ápice. Revista de Educación Cientifica, 2(2), 29-42.

Domènech-Casal J. (2019) Aprenentatge Basat en Projectes, Treballs pràctics $i$ Controvèrsies. 28 experiències $i$ reflexions per a ensenyar ciències. Rosa Sensat: Barcelona. 
Domènech-Casal J. (en edición) Contexto y modelo en el Aprendizaje Basado en Proyectos. Apuntes para el ámbito científico. Alambique, Didáctica de las Ciencias Experimentales, en edición.

Domènech-Casal J. y Lope S. (2015) Les Jornades de Controvèrsia Sòcio-Científica i Recerca i Innovació Responsables: Ciències per a qüestionar i canviar el món. Pensar,comprendre, decidir. Revista Ciències, 30, 29-31.

Duranti A. y Goodwin C. (Eds). (1992) Rethinking context: Language as an interactive phenomenon. Cambridge: Cambridge, University Press.

Ertmer P.A. y Simons K.D. (2005) Scaffolding teachers' efforts to implement problem-based learning. International Journal of Learning, 12(4), 319-328.

Garet M.S., Porter A.C., Desimone L., Birman B.F. y Yoon K.S. (2001) What makes professional development effective? Results from a national sample of teachers. American Educational Research Journal, 38(4), 915-945.

Gil D. y Vilches A. (2001) Una alfabetización científica para el siglo XXI. Obstáculos y propuestas de actuación. Investigación en la escuela 43, 27-37.

Gilbert J.K. (2006) On the nature of context in chemical education. International Journal of Science Education, 28(9), 957-976.

Grau R. (2009) Altres formes de fer ciència. Alternatives a l'aula de secundària. Rosa Sensat, Barcelona.

Henriksen D. (2014) Full STEAM Ahead: Creativity in Excellent STEM Teaching Practices. The STEAM Journal, 1(2), 15.

Hsieh H.F. y Shannon S.E. (2005) Three approaches to qualitative content analysis. Qualitative bealth research, 15(9), 1277-1288.

Hurd P.D. (1991) Why we must transform Science Education. Educational leadership, 49(2), 3335.

Jardine N. y Sibson R. (1971) Mathematical Taxonomy. Wiley, New York.

Johnson S.C. (1967) Hierarchical Clustering Schemes. Psychometrika, 32(3), 241-254.

Kilpatrick W.E. (1918) The Project Method: the use of the purposeful act in the educative process. Teachers college, Columbia University.

Kolstø S.D. (2001) Scientific Literacy for Citizenship: Tools for Dealing with the Science Dimension of Controversial Socioscientific Issues. Science Education, 85(3), 291-310.

Kortland J. (2007) Context-based science curricula: Exploring the didactical frictions between context and science content. ESERA. Malmö.

Larmer J., Mergendoller J. y Boss S. (2015) Setting the Standard for Project Based Learning: A Proven Approach to Rigorous Classroom Instruction. ASCD, Alexandria.

López V., Couso D., Simarro C., Garrido A., Grimalt-Álvaro C., Hernández M.I. y Pintó R. (2017) El papel de las TIC en la enseñanza de las ciencias en secundaria desde la perspectiva de la práctica científica. Enseñanza de las Ciencias, número Extraordinario 2017, 691-697.

Luft J.A., Roehrig G.H. y Patterson N.C. (2003) Contrasting landscapes: A comparison of the impact of different induction programs on beginning secondary science teachers' practices, beliefs, and experiences. Journal of Research in Science Teaching, 40(1), 77-97. 
Llewellyn D. (2005) Teaching High School Science through Inquiry: A case study approach. Corwin Press y NSTA press.

Marchán-Carvajal I., Palou L., Royo P. y Domènech-Casal J. (2017) Els contextos quotidians i els Estudis de Cas com a espai didàctic per a l'ensenyament de les Ciències basat en Projectes. Revista Ciències, 33, 8-14.

Margelí S., Perpinyà S., Monsergas M., Sol M. y Chiva S. (2017) La conjectura i la planificació. Aprenentatge de les matemàtiques mitjançant projectes. Revista Ciències, 33, 35-41.

Mesa M. (2000) La educación para el desarrollo: entre la caridad y la ciudadanía global. Papeles de Cuestiones Internacionales, 70, 11-26.

National Research Council (2014) STEM integration in K-12 education: Status, prospects, and an agenda for research. Washington, DC: The National Academies Press. doi: 10.17226/18612.

Pilot A. y Bulte A.M.W. (2006) Why Do You "Need to Know"? Context-based education. International Journal of Science Education 28 (9) 953 - 956.

Planella M., Vilches S., Font J., Grau R. y Gorriz, M. (2017) Espai, context i creativitat. Aprenentatge de les matemàtiques. Revista Ciències, 33, 42-48.

Pólya G. (1962) Mathematical Discovery: On Understanding, Learning and Teaching Problem Solving. New York: John Wiley y Son

Redondo M., Ruiz N., Sánchez S., Solé R., Calvet J. y Caño M. (2017) Context, Entorn i Servei: l'aprenentatge mitjançant projectes, de les àrees científiques a la Interdisciplinarietat. Revista Ciències, 33, 49-56.

Rocard M., Csermely P., Jorde D., Lenzen D., Walwerg-Heriksson H. y Hemmo V. (2007) Science Education Now: a new pedagogy for the future of Europe. Report for the European Comission. Disponible en línea: <http://ec.europa.eu/research/sciencesociety/document_library/pdf_06/report-rocard-on-science-education_en.pdf >.

Sanmartí N. (2016) Trabajo por proyectos: ¿Filosofía o metodología? Cuadernos de Pedagogía, 472, 44-46.

Sanmartí N., Burgoa B. y Nuño T. (2011) ¿Por qué el alumnado tiene dificultades para utilizar sus conocimientos escolares en situaciones cotidianas? Alambique, Didáctica de las Ciencias Experimentales, 67, 62-68.

Sanmartí N. y Márquez C. (2017) Aprendizaje de las ciencias basado en proyectos: del contexto a la acción. Ápice, Revista de Educación Científica, 1(1), 3-16.

Sadler T.D. (2009) Situated learning in science education: socio-scientific issues as contexts for practice. Studies in Science Education, 45(1), 1-42.

Schoenfeld A. (1992) Learning to think mathematically: problem solving, metacognition and sense making in mathematics. In: Handbook for Research on Mathematics Teaching and Learning. New York: Macmillan.

Smith T. M. y Ingersoll R. M. (2004) What are the effects of induction and mentoring on teacher turnover? American Educational Research Journal, 41(3), 681-714.

Simarro C. y Couso D. (2016) Análisis de una actividad tinkering en el marco de la educación STEM. Comunicación y Pedagogía 291-292, 65-7. 
Simarro C., López V., Cornellà P., Peracaula M., Niell M. y Estebanell M. (2016) Més enllà de la programació i la robótica educativa: el pensament ocmputacional en l'ensenyament STEAM a infantil i primària. Revista Ciències, 32, 38-46.

Touron J.L., Vergara Q., Arcas A. y Costa M. (2017) Els gèneres de comunicació científica i el laboratori com a espai didàctic per a l'ensenyament de les Ciències basat en Projectes. Revista Ciències, 33, 15-20.

Trujillo F. (2016) El diseño de proyectos y el currículo. Cuadernos de Pedagogía, 472, 66-69.

Josa Z., Pintó E., Serra C. y Feliu J. (2017) De l'electricitat a la fusta. Espais d'aprenentatge de la Tecnologia mitjançant projectes. Revista Ciències, 33, 21-28.

Vilches A., Gil D. y Cañal P. (2010) Educación para la sostenibilidad y educación ambiental. Investigación en la Escuela, 71, 5-15.

Villar J., Zurita S. y Giménez F. (2017) Apps i automatismes, oportunitats amb propòsit per a l'aprenentatge de la Tecnologia mitjançant projectes. Revista Ciències, 33, 29-34.

Wang H.H., Moore T.J., Roehrig G.H., Park M.S. (2011) STEM Integration: Teacher Perceptions and Practice. Journal of Pre-College Engineering Education Research, 1(2) 1-13.

Wilson S. (2009) Teacher quality: Education policy white paper. National Academy of Education. Washington, DC.

Windschitl M., Thompson J. y Braaten M. (2008) Beyond the scientific method: Model-based inquiry as a new paradigm of preference for school science investigations. Science Education, 92(5), 941-967.

Wing J.M. (2006) Computational Thinking. Communications of the ACM, 49(3), 33-35.

Yager R.E. (Ed.) (1996) Science/Technology/Society as a Reform in Science Education. New York: Exelsior Editions, State University of New York Press. 